\title{
Alcohol Withdrawal and Flumazenil: Not for the Faint of Heart
}

\author{
Lewis S. Nelson
}

Published online: 5 March 2014

(C) American College of Medical Toxicology 2014

It is almost paradoxical that medical toxicologists are frequently involved with the management of patients with alcohol withdrawal, given that this syndrome is due to the absence, not the presence of a toxin. Because of our understanding of the pharmacologic underpinnings of both the syndrome and its treatment, medical toxicologists typically recommend a goal-directed treatment of this disease at its early stages, with full understanding that this carries consequences, such as prolonged sedation, that are easily offset by the improvement in outcome [1]. Our experience breeds expertise that guides the management of this complex clinical problem not just in the ED, but during the patient's entire course in the hospital. This is well illustrated in the paper published in this issue by Moore et al., which looked at the concluding steps in the treatment of such patients [2].

The alcohol withdrawal syndrome (AWS), the constellation of signs and symptoms that alcoholics suffer upon cessation or reduction of alcohol intake, remains to be a century-old clinical challenge faced by health-care providers. Despite the millennia-long use of ethanol, we have a remarkably limited understanding of the pathophysiology of both drunkenness and AWS. Until as recently as the 1950s, the cause of the tremors and delirium associated with ethanol discontinuation was actually believed to be the excessive alcohol consumption itself along with nutritional deficiency, not the act of cessation.

In 1953, Victor and Adams observed in exquisite detail the clinical consequences of abstinence in 206 alcoholic patients hospitalized at Boston City Hospital [3]. This was followed by the "landmark," and quite unethical by today's standards, study in 1955 by Isbell that supported the burgeoning understanding that there was a dose-response relationship of ethanol to the severity of AWS [4]. In this study, ten prisoners, who

L. S. Nelson $(\bowtie)$

New York University School of Medicine, New York, NY, USA

e-mail: lewis.nelson@nyumc.org were all former morphine addicts, were assigned to consume large amounts of alcohol for periods of time varying from 1 week to several months. Consumption was abruptly discontinued at the end of these time periods, and the subjects were monitored for signs of AWS. Not surprising to any current reader, but novel in 1955, the longer the period of consumption, the more severe the withdrawal. Subsequently, in 1964, the work by Mendelson and Mello provided the first controlled experimental evidence that withdrawal was directly related to drinking cessation [5].

Despite a lack of understanding of the reasons for withdrawal, as early as the turn of the twentieth century, alcohollike drugs, such as paraldehyde and chloral hydrate, were introduced into clinical practice for its management. In the 1950s, phenothiazines were introduced with checkered success, followed by the barbiturates and benzodiazepines through the 1960s and 1970s. Some centers used, and some amazingly continue to use, ethanol either orally or by infusion to prevent and treat AWS, particularly in trauma patients [6]. In the 1990s, the approach to AWS underwent a paradigm shift, with the acceptance of symptom-triggered therapy [7].

It is now clear that AWS is best treated by administering benzodiazepines using a symptom-triggered approach, meaning the next dose is administered when the patient begins to feel or display recrudescent AWS (such as a rising CIWA, RASS, or Riker score). Using this approach, a reduction in the total dose of benzodiazepines required and the length of stay have been demonstrated in controlled trials [8]. However, the responsiveness to individual doses of benzodiazepine varies widely among patients [1]. The reasons for this are unknown, but there may be, in part, a genetic determinant [9]. Regardless of the reason, the relative resistance to benzodiazepines displayed by some patients has led to the administration of escalating, and sometimes massive, doses of drug, in what amounts to a pharmacologic game of cat and mouse [10]. It is not uncommon for a patient with AWS to receive several 
hundred milligrams of diazepam (or equivalent) over the course of 3 or 4 days. They are in turn able to handle such large doses due presumably to the development of acute benzodiazepines tolerance on top of a baseline of ethanol tolerance.

While benzodiazepines (and other GABAergics) are the mainstay of treatment [11], they are clearly an imperfect option. This is highlighted by the fact that, at doses necessary for symptom reduction, benzodiazepines produce sedation and delirium rather than make the patient "normal." Looked at another way, every morning upon awakening, an alcoholdependent person requires what amounts to an "eye-opener" to stave off the effects of early signs of AWS. This produces a numerically though not clinically intoxicated person, who is neither sedate nor confused. Thus, the cross-tolerance of GABAergics with ethanol turns off the major features of AWS, prevents progression to DTs, and stops seizures, all in a safe and relatively predictable manner (notwithstanding the dose variation), though at the expense of sedation and delirium. In a world of perfect pharmacokinetics, administration of a dose of diazepam sufficient to induce a sedate and stable patient could be allowed to "auto taper" over the next several dose, perhaps with a little "topping off" as needed $[1,12]$.

Indeed, with chronic alcohol exposure, there is a switch in GABAA receptor subtype (from alpha 1 to alpha 4) [13], producing a receptor that is less sensitive to the depressant effects of ethanol (a clinical benefit leading to tolerance) but also appears proportionally less sensitive to benzodiazepines (a therapeutic liability). Alternatively, it has become increasingly clear that an over-expression of glutamatergic neurotransmission may account for some of the shortcomings of GABAergic therapy [14]. It also explains the relative success of managing AWS with medications that have NMDA receptor antagonist effects, such as propofol [15]. In addition, it may explain why the eye-opener dose of ethanol, which blocks the glycine-binding site of the NMDA receptor, results in a clear sensorium, while benzodiazepines fail to affect a reduction in glutamatergic excitation and the resulting delirium. These changes appear to be due to alterations induced by ethanol in neurosteroids, which are positive allosteric modulators of the GABAA receptor and a myriad of other neurotransmitters such as dopamine [16, 17].

What happens neurochemically during detoxification remains unclear. For example, it is not known if the receptor conformations reset to baseline or if new counter-regulatory mechanisms are engaged to counteract the effects of the altered receptors. What is clear is that at some point, usually at around day 3 or 4 post-cessation, the dependence on, and likely tolerance to, ethanol moderates sufficiently, and the AWS wanes.

However, what is left is the lingering effect of the benzodiazepine, which is, out of necessity, generally given at doses in excess of what is pharmacologically required. This occurs because the half-lives of all of the benzodiazepines, particularly following large and repetitive doses, are quite long and appear to span the time frame needed for the AWS to abate. We favor this because in concept (as mentioned above), it allows the withdrawal syndrome to subside as the benzodiazepine effects wane, in near-perfect harmony.

So, that brings us back to the study by Moore et al. in this issue. This represents an interesting new direction in the management of the patients who appear to have resistant AWS. After being given doses of benzodiazepines that we have all grown accustomed to administering, their patients, as expected, had a lingering delirium even after the clinical signs of AWS are resolved. In this single-center study, when the patient was deemed to no longer have AWS, somewhere around day 5 on average, they were administered flumazenil to reverse their benzodiazepine stupor. This raises a few interesting questions.

First, is this effective? In nearly three quarters of their patients, there was objective evidence of improvement, although it was only really prominent in over-sedated patients. Those patients with agitated delirium still responded however, which may be akin to reversal of the paradoxical release effects of benzodiazepines seen commonly in young and old patients.

Is it safe? The thought of giving flumazenil to this group of patients may seem anathema to many, given the risks associated with rapid benzodiazepine reversal in dependent patients and the propensity of AWS patients to seize. However, the authors found little in the way of adverse effects.... no serious agitation, no seizures, apparently no need for additional meds to treat a complication. However, Victor noted that virtually all AWS seizures occur within $48 \mathrm{~h}$, and the time between the first and last seizure is less than $6 \mathrm{~h}$ in over $90 \%[3,18]$. Furthermore, the argument can be made that these patients are not yet deeply dependent on benzodiazepines since they had only been on this class of medication for an average of 5 days, hardly enough time for concern.

The most interesting question to ponder is what is going on at the receptor level. These patients are all heavy users of and dependent on alcohol. Yet, they become sedate after benzodiazepines, suggesting that benzodiazepines work differently than ethanol at the GABAA receptor. By working through this distinct neuroinhibitory mechanism, the benzodiazepines may simply provide the neurons a few days to "lose" their tolerance to alcohol. The potential for two simultaneous processes (benzodiazepine sedation and alcohol recovery), rather than simple replacement of one sedative for another, is intriguing.

This is a non-controlled, retrospective study with semiobjective endpoints and variable monitoring and assessment patterns, restricting external validity. Since there is no control group, it remains unclear if we are witnessing an analeptic effect of flumazenil, although its beneficial effects seem to be bidirectional (treating both hyperactive and hypoactive 
deliriums). In addition, the frequent use of other medications such as clonidine, opioids, and antipsychotics, which are rarely necessary, may blur the results for many readers. Given these limitations, we may not be ready to recommend this to our colleagues. Some of us, however, may believe the results and be willing to try a small flumazenil dose, $0.3 \mathrm{mg}$ in the study, in some of our own patients. This may not be for the faint of heart, but then again, if medical toxicologists cannot venture into uncharted pharmacologic territory.... who can?

Conflict of Interest No conflicts of interest to report.

\section{References}

1. Gold JA, Rimal B, Nolan A, Nelson LS (2007) A strategy of escalating doses of benzodiazepines and phenobarbital administration reduces the need for mechanical ventilation in delirium tremens. Crit Care Med 35:724-730. doi:10.1097/01.CCM.0000256841. 28351.80

2. Moore PW, Donovan JW, Burkhart KK et al (2014) Safety and Efficacy of Flumazenil for Reversal of Iatrogenic BenzodiazepineAssociated Delirium Toxicity During Treatment of Alcohol Withdrawal, a Retrospective Review at One Center. J. Med. Toxicol. doi: 10.1007/s13181-014-0391-6

3. Victor M, Adams RD (1953) The effect of alcohol on the nervous system. Res Publ Assoc Res Nerv Ment Dis 32:526-573

4. Isbell H, Fraser HF, Wikler A et al (1955) An experimental study of the etiology of rum fits and delirium tremens. Q J Stud Alcohol 16:1-33

5. Mendelson JH, Mello NK (1964) Metabolism of C-14 ethanol and behavioral adaptation of alcoholics during experimentally induced intoxication. Trans Am Neurol Assoc 89:133-135

6. Weinberg JA, Magnotti LJ, Fischer PE et al (2008) Comparison of intravenous ethanol versus diazepam for alcohol withdrawal prophylaxis in the trauma ICU: results of a randomized trial. J Trauma 64: 99-104. doi:10.1097/TA.0b013e31815eb12a
7. Saitz R, Mayo-Smith MF, Roberts MS et al (1994) Individualized treatment for alcohol withdrawal. A randomized double-blind controlled trial. JAMA 272:519-523

8. Muzyk AJ, Leung JG, Nelson S et al (2013) The role of diazepam loading for the treatment of alcohol withdrawal syndrome in hospitalized patients. Am J Addict 22:113-118. doi:10.1111/j.1521-0391. 2013.00307.x

9. Chan GM, Hoffman RS, Gold JA et al (2009) Racial variations in the incidence of severe alcohol withdrawal. J Med Toxicol 5: $8-14$

10. Hack JB, Hoffmann RS, Nelson LS (2006) Resistant alcohol withdrawal: does an unexpectedly large sedative requirement identify these patients early? J Med Toxicol 2:55-60

11. Amato L, Minozzi S, Davoli M (2011) Efficacy and safety of pharmacological interventions for the treatment of the alcohol withdrawal syndrome. Cochrane Database Syst Rev, Issue 6. Art. No.: CD008537. doi:10.1002/14651858.CD008537.pub2

12. Maldonado JR, Nguyen LH, Schader EM, Brooks JO (2012) Benzodiazepine loading versus symptom-triggered treatment of alcohol withdrawal: a prospective, randomized clinical trial. Gen Hosp Psychiatry 34:611-617. doi:10.1016/j.genhosppsych.2012.06.016

13. Kumar S, Porcu P, Werner DF et al (2009) The role of GABA(A) receptors in the acute and chronic effects of ethanol: a decade of progress. Psychopharmacology 205:529-564. doi:10.1007/s00213009-1562-z

14. Krupitsky EM, Rudenko AA, Burakov AM et al (2007) Antiglutamatergic strategies for ethanol detoxification: comparison with placebo and diazepam. Alcohol Clin Exp Res 31:604-611. doi:10.1111/j.1530-0277.2007.00344.x

15. Zhang H, Wang W, Gao W et al (2009) Effect of propofol on the levels of neurotransmitters in normal human brain: a magnetic resonance spectroscopy study. Neurosci Lett 467:247-251. doi:10.1016/ j.neulet.2009.10.052

16. Roberto M, Gilpin NW, Siggins GR (2012) The central amygdala and alcohol: role of $\gamma$-aminobutyric acid, glutamate, and neuropeptides. Cold Spring Harb Perspect Med 2:a012195. doi:10.1101/ cshperspect.a012195

17. Follesa P, Biggio F, Talani G et al (2006) Neurosteroids, GABAA receptors, and ethanol dependence. Psychopharmacology 186:267280. doi:10.1007/s00213-005-0126-0

18. Victor M, Brausch C (1967) The role of abstinence in the genesis of alcoholic epilepsy. Epilepsia 8:1-20 